\title{
Volatility Analysis of Nepalese Stock Market
}

\author{
Surya Bahadur G.C.
}

\begin{abstract}
Modeling and forecasting volatility of capital markets has been important area of inquiry and research in financial economics with the recognition of time-varying volatility, volatility clusturing, and asymmetric response of volatility to market movements. Given the anticipated growth of the Nepalese stock market and increasing interest of investors towards investment in Nepalese stock market, it is important to understand the pattern of stock market volatility. In the paper, the volatility of the Nepalese stock market is modeled using daily return series consisting of 1297 observations from July 2003 to Feb 2009 and different classes of estimators and volatility models. The results indicate that the most appropriate model for volatility modeling in Nepalese market, where no significant asymmetry in the conditional volatility of returns was captured, is $\operatorname{GARCH}(1,1)$. The study revealed strong evidence of time-varying volatility, a tendency of the periods of high and low volatility to cluster and a high persistence and predictability of volatility in the Nepalese stock market.
\end{abstract}

Key words : Conditional heteroskedasticity, ARCH, GARCH, volatility clustering, leverage effect, Nepalese Stock Market

\section{Introduction}

Stock prices volatility has received a great attention from both academicians and practitioners over the last two decades because it can be used as a measure of risk in financial markets. Over recent years, there has been a growth in interest in the modelling of time-varying stock return volatility. Many economic models assume that the variance, as a measure of uncertainty, is constant through time. However, empirical evidence rejects this assumption. Economic time series have been found to exhibit periods of unusually large volatility followed by periods of relative tranquility (Engle, 1982). In such circumstances, the assumption of constant variance (homoskedasticity) is inappropriate (Nelson,1991). The time series are found to depend on their own past value (autoregressive), depend on past information (conditional) and exhibit non-constant variance (heteroskedasticity). It has been found that the stock market volatility changes with time (i.e., it is "time-varying") and also exhibits positive serial correlation or "volatility clustering". Large changes tend to be followed by large changes and small changes tend to be followed by small changes, which mean that volatility clustering is observed in financial returns data. This implies that the 76 
changes are non-random. When volatility clusters, the random walk hypothesis is refuted (Mandelbrot, 1963).

Moreover, the volatility of returns can be characterized as a long-memory process as it tends to persist. the technical term given to this behavior is autoregressive conditional heteroskedasticity (ARCH). In a seminal paper, Engle (1982), for the first time, proposed to model time-varying conditional variance with the ARCH process that uses past disturbances to model the variances of the series and allows the variance of the error term to vary over time. Bollerslev (1986) generalized the $\mathrm{ARCH}$ process by allowing the conditional variance to be a function of prior period's squared errors as well as its past conditional variances. Following the introduction of models of ARCH by Engle (1982) and their generalization by Bollerslev (1986), there have been numerous refinements of the approach to modelling conditional volatility to better capture the stylized characteristics of the data. Empirically, the family of GARCH (generalized ARCH) models has been very successful in describing the financial data. ARCH and GARCH models treat heteroskedasticity as a variance to be modeled. Of these models, the GARCH $(1,1)$ is often considered by most investigators to be an excellent model for estimating conditional volatility for a wide range of financial data (Bollerslev, Ray and Kenneth, 1992).

Financial time series often exhibit leptokurtosis, which means that the distribution of their returns is fat-tailed (i.e. relative high probability for extreme values) Mandelbrot (1963), Fama (1965), Laurent and Peters (2002). Moreover, changes in stock prices tend to be negatively correlated with changes in volatility. In financial data, the asymmetry is usually, such that negative shocks cause higher volatility in the near future than the positive shocks, which is called "leverage effect" (Black, 1976; Nelson, 1991). Though, in most of the cases, the ARCH and GARCH models are apparently successful in estimating and forecasting the volatility of the financial time series data, they cannot capture some of the important features of the data. The most interesting feature not addressed by these models is the "leverage effect" where the conditional variance tends to respond asymmetrically to positive and negative shocks in returns. They fail to capture the fat-tail property of financial data. This has lead to the use of non-normal distributions (Student-t, Generalized Error Distribution and Skewed Student- $t$ ), within many nonlinear extensions of the GARCH model which have been proposed. Such as the Exponential GARCH (EGARCH) of Nelson (1991) the so-called GJR model of Glosten, Jagannathan, and Runkle (1993) and the Asymmetric Power ARCH (APARCH) of Ding, Granger, and Engle (1993), to better model the fat-tailed (the excess kurtosis), skewness and leverage effect characteristics .

Although, recently, a few studies on stock market volatility using ARCH family models have been carried out on emerging markets including India (Karmakar, 2005, Pandey, 
2005), surprisingly enough, there have been quite a few studies focusing on small capital markets. The focus of the study is to model conditional volatility in an effort to capture the salient features of stock market volatility and investigate whether there is any leverage effect in the Nepalese capital market.

\section{Data and Review of Volatility Models}

The data set comprises of the daily NEPSE Index return from $17^{\text {th }}$ July, 2003 to $11^{\text {th }} \mathrm{Feb}, 2009$ with a total number of observations of 1297 . The data covers the periods of varying volatility patterns observed in the Nepalese market. The daily return series will be generated as follows:

$$
\mathrm{R}_{\mathrm{SI}, \mathrm{t}}=(100) *\left(\log \left(\mathrm{R}_{\mathrm{SI}, \mathrm{t}}-\mathrm{R}_{\mathrm{SI}, \mathrm{t}-\mathrm{t}}\right)\right)
$$

Where $\mathrm{R}_{\mathrm{SI}, \mathrm{t}}$ represents the closing value of the stock index on the day $\mathrm{t}$. The return series is therefore continuously compounded daily returns expressed as a percentage. Under the ARCH model, the 'autocorrelation in volatility' is modeled by first estimating the conditional mean and variance. A time series for index $R_{t}$ can be thought of as the sum of a predictable and an unpredictable component:

$$
R_{t}=E\left(R_{t} / R_{t-1}\right)+u_{t} \ldots \quad \ldots \quad \ldots \quad \text { (ii) }
$$

The conditional variance is given by:

$$
\mathrm{V}\left(\mathrm{R}_{\mathrm{t}} \mid \mathrm{R}_{\mathrm{t}-1}\right)=\mathrm{E}\left(\mathrm{R}_{\mathrm{t}}-\mathrm{E}\left(\mathrm{R}_{\mathrm{t}} \mid \mathrm{R}_{\mathrm{t}-1}\right)\right)^{2}=\mathrm{E}\left(\mathrm{R}_{\mathrm{t}-1}+\mathrm{e}_{\mathrm{t}}-\mathrm{E}\left(\mathrm{R}_{\mathrm{t}} \mid \mathrm{R}_{\mathrm{t}-1}\right)\right)^{2}=\sigma^{2} \ldots \ldots \quad \ldots
$$

Under the ARCH model the 'autocorrelation in volatility' is modeled by allowing the conditional variance of the error term, $\sigma^{2}$ to depend on the immediately previous value of the squared error:

$$
\sigma_{\mathrm{t}=}^{2} \alpha_{0}+\alpha_{\mathrm{t}} \mathrm{u}_{\mathrm{t}-1}^{2} \quad \cdots \quad \cdots \quad \cdots
$$

The above model is known as $\mathrm{ARCH}(1)$, since the conditional variance depends on only one lagged squared error term. The model can be extended to the general case where the error variance depends on q lags of squared errors, which would be known as an $\operatorname{ARCH}(q)$ model:

$$
\sigma_{\mathrm{t}=}^{2} \alpha_{0}+\alpha_{1} \mathrm{u}_{\mathrm{t}-1}^{2}+\alpha_{2} \mathrm{u}_{\mathrm{t}-2}^{2}+\ldots \ldots \ldots \ldots+\alpha_{\mathrm{q}} \mathrm{u}_{\mathrm{t}-\mathrm{q}}^{2} \ldots \ldots \ldots
$$

The appropriate lag length ' $\mathrm{q}$ ' is determined by using AIC and SIC. The GARCH model allows the conditional variance to be dependent upon previous own lags, so that the conditional variance equation in the simplest case is :

$$
\sigma_{\mathrm{t}=}^{2} \alpha_{0}+\alpha_{1} \mathrm{u}_{\mathrm{t}-1}^{2}+\beta_{1} \sigma_{\mathrm{t}-1}^{2} \quad \cdots \quad \cdots \quad \cdots
$$

This is the $\operatorname{GARCH}(1,1)$ model, $\sigma_{\mathrm{t}}^{2}$ is known as the conditional variance since it is a one-period ahead estimate for the variance calculated based on any past information thought relevant. The GARCH is more parsimonious and avoids data over-fitting as compared to ARCH. The GARCH $(1,1)$ model can be extended to a GARCH(p,q) formulation, where 78 
the current conditional variance is parameterized to depend upon q lags of the squared error and $\mathrm{p}$ lags of the conditional variance

$$
\sigma_{t}^{2}=\alpha_{0}+\sum^{q} \alpha_{i} u_{t-i}^{2}+\sum^{p} \beta_{j} \sigma_{t-j}^{2}
$$

One of the primary restrictions of GAR $G H$ models is that they enforce a symmetric response of volatility to positive and negative shocks. However, it has been argued that a negative shock to financial time series is likely to cause volatility to rise by more than a positive shock of the same magnitude. A term referred to as leverage effect. The study will use two popular asymmetric GARCH models to test for the presence of leverage effect in Nepalese data: the GJR model and the exponential GARCH (EGARCH) model. The GJR model or Threshold GARCH (TGARCH) is a simple extension of GARCH with an additional term added to account for possible asymmetries (Knight and Satchell, 2003). The conditional variance is now given by:

$$
\sigma_{\mathrm{t}=}^{2} \alpha_{0}+\alpha_{1} \mathrm{u}_{\mathrm{t}-1}^{2}+\beta_{1} \sigma_{\mathrm{t}-1}^{2}+\gamma \mathrm{u}_{\mathrm{t}-1}^{2} \mathrm{I}_{\mathrm{t}-1} \ldots \ldots
$$

Where $\mathrm{I}_{\mathrm{t}-1}=1$ if $\mathrm{u}_{\mathrm{t}-1}<0$ or zero otherwise. For a leverage effect, we would see $\gamma>0$. The EGARCH model expresses the conditional variance as follows (Brooks, 2004):

$$
\ln \left(\sigma^{2} t\right)=\alpha_{0}+\alpha_{1} \ln \left(\sigma^{2}{ }_{t-1}\right)+\beta_{1} \frac{u_{t-1}}{\sqrt{\sigma^{2} t-1}}+\gamma\left[\frac{\left|u_{t-1}\right|}{\sqrt{\sigma_{t-1}^{2}}}-\sqrt{\frac{2}{\pi}}\right]
$$

Asymmetries are allowed for under the EGARCH model, since if the relationship between the volatility and returns is negative, $\beta_{1}$ will be negative.

\section{Descriptive Statistics of Nepse Return Series}

The index has a large difference between its maximum and minimum returns. The standard deviation is also high indicating a high level of fluctuations of the NEPSE daily return. There is also evidence of negative skewness, which means that the left tail is particularly extreme, and indication that the NEPSE has non-symmetric returns. NEPSE's returns are leptokurtic or fat-tailed, given its large kurtosis.

Figure 1: Descriptive Statistics of NEPSE Index Return Series

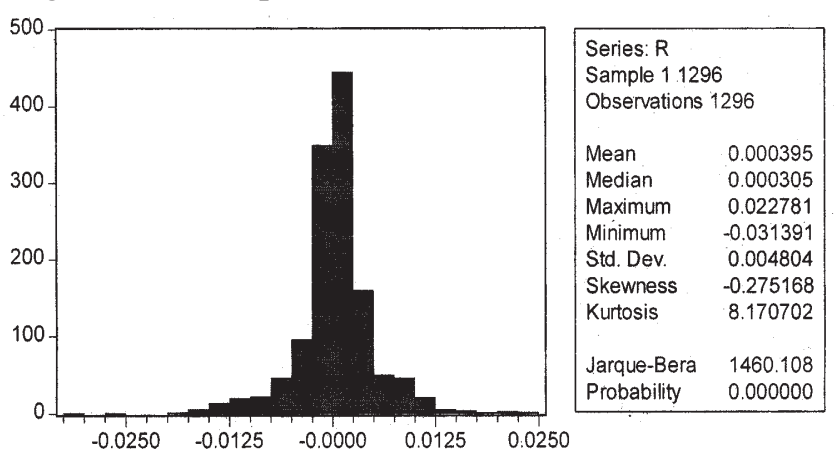


The negative skewness implies that the return distributions of the shares traded in our markets have a higher probability of earning negative returns. The market average effective annual return for the study period is 15.27 percentage. The historical equity risk premium is around 10 percent. The Jarque-Bera test rejects the null hypothesis of normality providing the evidence that the return series is not normally distributed.

\section{Volatility Clusturing and Modelling Volatility}

Figure 2 exhibits the return series of the NEPSE Index for the period $17^{\text {th }}$ July, 2003 to $11^{\text {th }}$ Feb, 2009. There are stretches of time where the volatility is relatively high and relatively low which suggests an apparent volatility clusturing in some periods. Statistically, volatility clusturing implies a strong autocorrelation in returns. Volatility clusturing describes the tendency of large changes in assets prices (of either sign) to follow large changes and vice versa. In other words, the current level of volatility tends to be positively correlated with its level during the immediately preceding periods.

\section{Figure 2: Volaltility Clusturing of Daily Returns of NEPSE Index}

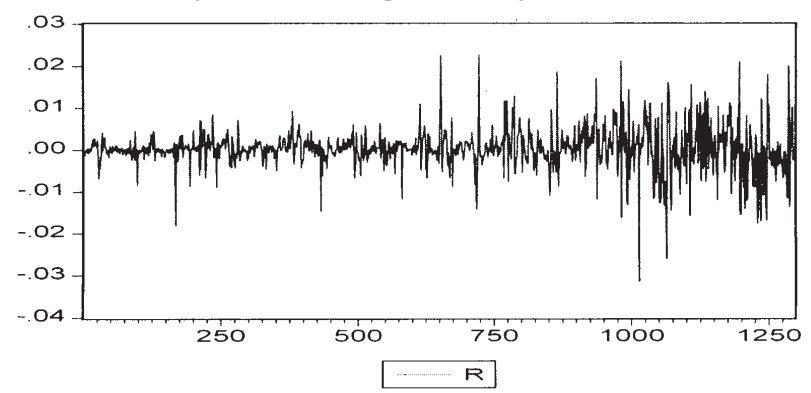

The above figure shows that the volatility in Nepalese market has increased in recent periods. There appears to have been a prolonged period of relative tranquility in the market during the early study period, evidenced by only relatively small positive and negative returns. The series exhibits volatility clusturing and time-varying characteristics of volatility. The last two columns reported in the correlogram shown in Appendix II are the Ljung-Box Q-statistics and their p-values. The Q-statistic at lag $\mathrm{k}$ is a test statistic for the null hypothesis that there is no autocorrelation up to order k. (lag length). The values of Q statistics, ACF and PACF suggest the presence of autocorrelation and hence volatility clusturing in return series of the NEPSE index. We can observe that the index shows evidence of ARCH effects judging from the significant autocorrelation coefficients. The autocorrelation in the series dies out after 28 lags. The characteristics of the NEPSE return series are consistent with other financial times series. To sum up, the analysis indicates that the daily return series of the index is non-normal and exhibits 'ARCH effect'. 
The ARCH(4), GARCH (1,1), EGARCH(1,1), TGARCH(1,1) models are estimated for the NEPSE returns series to choose the volatility model that best models the conditional volatility of the return series. A test for the presence of ARCH in the residuals is calculated by regressing the squared residuals on a constant and $\mathrm{p}$ lags. The correct number of lags in the model have been selected using AIC and SIC information criterion. The test can also be thought of as a test for autocorrelation in the squared residuals.

Table 1: Model Output of ARCH(4) Test

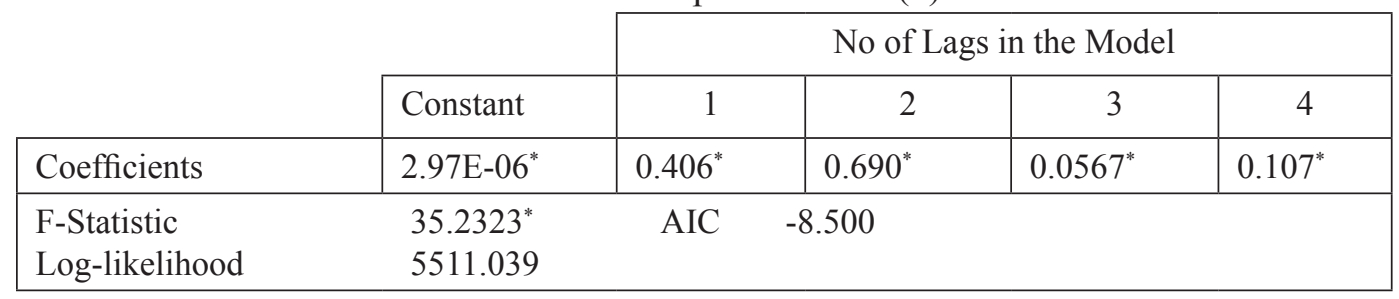

${ }^{*}$ Significant at $1 \%$ level of significance

Both the F-statistic and the LM-statistic are very significant, suggesting the presence of ARCH in the NEPSE index returns. The non-negativity constraints of the coefficients have not been violated. ARCH models provide a framework for the analysis and development of time series models of volatility. However, most recent empirical studies use GARCH model than $\mathrm{ARCH}$ as it is more parsimonious and avoids overfitting.

Table 2: Estimated Volatility Models

\begin{tabular}{cccccc}
\hline Models & RW & GARCH & TGARCH & EGARCH & PARCH \\
\hline Mean Equation & & & & & \\
\hline$\mu$ & $0.0002^{* *}$ & $7.32 \mathrm{E}-05$ & $0.00011^{* *}$ & $3.16 \mathrm{E}-05$ & $0.0001^{*}$ \\
$\alpha$ & $0.37^{*}$ & $0.3298^{*}$ & $0.327^{*}$ & $0.344^{*}$ & 0.359 \\
\hline Variance Equation & & & & & \\
\hline$\alpha_{0}$ & - & $1.06 \mathrm{E}-06^{*}$ & $1.02 \mathrm{E}-06^{*}$ & $-1.75^{*}$ & $0.0007^{* *}$ \\
$\alpha_{1}$ & - & $0.417^{*}$ & $0.605^{*}$ & $0.882^{*}$ & $0.336^{* *}$ \\
$\beta_{1}$ & - & $0.55^{*}$ & $0.563^{*}$ & $0.051^{*}$ & $-0.081^{* *}$ \\
$\gamma$ & - & - & $-0.0131^{* *}$ & $0.619^{*}$ & $0.681^{*}$ \\
$\theta$ & - & - & - & - & $0.860^{*}$ \\
Adj R & & & & & \\
F-statistic & $213.59^{*}$ & $51.63^{*}$ & $41.41^{*}$ & $41.41^{*}$ & $35.22^{*}$ \\
Jarque-Bera & 3238 & 4301 & 4016 & 3015 & 3673
\end{tabular}




\begin{tabular}{lccccc}
\hline AIC & -7.98 & -8.487 & -8.48 & -8.514 & -8.51 \\
LL & 5174 & 5500 & 5502 & 5519 & 5519 \\
No of Obs. & 1297 & 1297 & 1297 & 1297 & 1297 \\
\hline
\end{tabular}

* and ** indicates that the coefficient values are significant at $1 \%$ and $5 \%$ level of significance respectively.

\section{Results and Discussion}

The results of estimation and statistical verification of the $\mathrm{RW}, \operatorname{GARCH}(1,1)$, TGARCH(1,1), EGARCH(1,1) and PARCH(1,1) models are shown in Table 2. The $\operatorname{AR} 1(\alpha)$ parameters in the mean equation are significant in all estimated models except the PARCH model. The ARCH $\left(\alpha_{1}\right)$ and GARCH $\left(\beta_{1}\right)$ terms are positive and significant in all estimations. The sum of ARCH and GARCH coefficients $(\alpha+\beta)$ is very close to one, indicating that the volatility shock are very persistent. It is an indication of a convariance stationary model with a high degree of persistence; and long memory in the conditional variance.

The parameter estimates of all the GARCH models in table 2 show that the coefficients of th conditional variance equation, $\alpha_{1}$ and $\beta_{1}$ are significant at one percent level of significance implying a strong support for the presence of ARCH and GARCH effects. Also, as is typical of GARCH model estimates for financial asset returns data, the sum of the coefficients is very close to unity. This implies that shocks to the conditional variance will be highly persistent. It implies that a large positive or negative return will lead future forecasts of the variance to be high for a protracted period. In $\operatorname{GARCH}(1,1)$ model, $\alpha+\beta=0.967$ is also an estimation of the rate at which the response function declays on daily basis. Since the rate is high, the response function to shock is likely to die slowly. It means new shocks will have influence in returns for a longer period. In such markets old information effect on securities prices decays very slowly. The findings is consitent with Magnus and Fosu (2006). For TGARCH models the persistence in volatility is very long and explosive suggestive of an integrated process. The asymmetric (leverage) effect captured by the parameter estimate $\gamma$ which is less than zero suggesting absence of leverage effect. The asymmetry term $\beta_{1}$ for EGARCH model is positive which also indicates absence of leverage effect. The absence of asymmetry or leverage effect is further supported by the output of the PARCH model.

The results of diagnostic tests show that the GARCH models are correctly specified. Overall, using the minimum AIC, maximum LL values as model selection criteria, the GARCH $(1,1)$ is the preferred model. The absence of leverage or asymmetric effects 
indicates that the $\operatorname{GARCH}(1,1)$ model best models the volatility of the NEPSE return series.

\section{Conclusions}

The study found the distribution of the daily return series for the Nepalese stock market to be leptokurtic, non-normal and exhibiting significant time dependencies. The conditional volatility of the NEPSE return series was modeled using a random walk model, a non-linear GARCH(1,1) model and threee asymmetric models GJR model, EGARCH( 1,1$)$ and PARCH $(1,1)$. The study found that the NEPSE Index return series exhibits stylized characteristics as supported by empirical evidence in different studies such as volatility clusturing, time-varying conditional heteroskedasticity, and leptokurtosis. However, the asymmetric leverage effect as evidenced on various studies in advanced stock markets was not detected in NEPSE index return series. The $\operatorname{GARCH}(1,1)$ was found to be the appropriate model for volatility forecasting in Nepalese stock market. The parameter estimates of the GARCH models suggest a high degree of persistence in conditional volatility of stock returns. The evidence of high volatility persistence and long memory in the GARCH process indicate that integrated GARCH model might be a better model for volatility analysis of the NEPSE data series. The study revealed strong evidence of timevarying volatility, a tendency of the periods of high and low volatility to cluster and a high persistence and predictability of volatility in the Nepalese stock market.

\section{References}

Black, F. (1976). "Studies of Stock Market Volatility Changes," Proceedings of the American Statistical Association, Business and Economic Statistics Section, 177-181.

Bollerslev, T. (1986). "Generalized Autoregressive Conditional Heteroscedasticity", Journal of Econometrics, Vol- 31, pp. 307-327.

Bollerslev, T., Chou, R., and Kroner, K. (1992). “ARCH Modeling in Finance”, Journal of Econometrics, Vol- 52, pp. 5-59.

Bollerslev, T, Engle, R.F., and Nelson, D.B. (1994). “ARCH Models”, Handbook of Econometrics, Vol- 4, pp. 2959-3038.

Brooks, C. (2004). Introductory Econometrics for Finance. Cambridge University Press.

Engle, R. F. (1982). "Autoregressive Conditional Heteroscedasticity with Estimates of the Variance of United Kingdom Inflation”, Econometrica, Vol- 50, pp. 987-1007.

Engle, R F and Granger, C. (1993). "Modelling the Persistence of Conditional Variances", Econometric Reviews, Vol- 5, pp. 1-50.

Fama, E F. (1965). "The Behavior of Stock Market Prices", Journal of Business, Vol-38, pp. 34-105.

Glosten, L.R., Jagannathan R., and Runkle, D.E. (1993). "On the Relation Between Expected Value and the Volatility of the Nominal Excess Returns on Stocks", Journal of Finance, Vol- 48:5, pp. 1779-801.

Karmakar, M. (2005). "Modelling Conditional Volatility of the Indian Stock Markets", Vikalpa: The Journal for Decision Makers, Vol- 30, pp. 21-37.

Knight, J.L. and Satchell, S. (2003). Forecasting Volatility in the Financial Markets, $2^{\text {nd }}$ Ed. Singapore: Wiley and Sons Ltd. 
Laurent, S. and Peters, J. (2002). “A tutorial for GARCH 2.3, a Complete Ox Packages for Estimating and Forecasting ARCH Models."

Mandelbrot, B. (1963). "The Variation of Certain Speculative Prices", Journal of Business, Vol- 36, pp. 394419.

Magnus, J.F. and Fosu, O.E.(2006). "Modelling and Forecasting Volatility of Returns on the Ghana Stock Exchange Using GARCH Models", American Journal of Applied Sciences, Vol 3(10).

Nelson, D. B. (1991). "Conditional Heteroscedasticity in Asset Returns: A New Approach", Econometrica, Vol- 59:2, pp. 347-70.

Pandy, A. (2005). "Volatility Models and their Performance in Indian Capital Markets", Vikalpa: The Journal for Decision Makers, Vol- 30, pp. 27-45.

\section{Appendix I: Movement in NEPSE Index from July 2003 to Feb 2009}

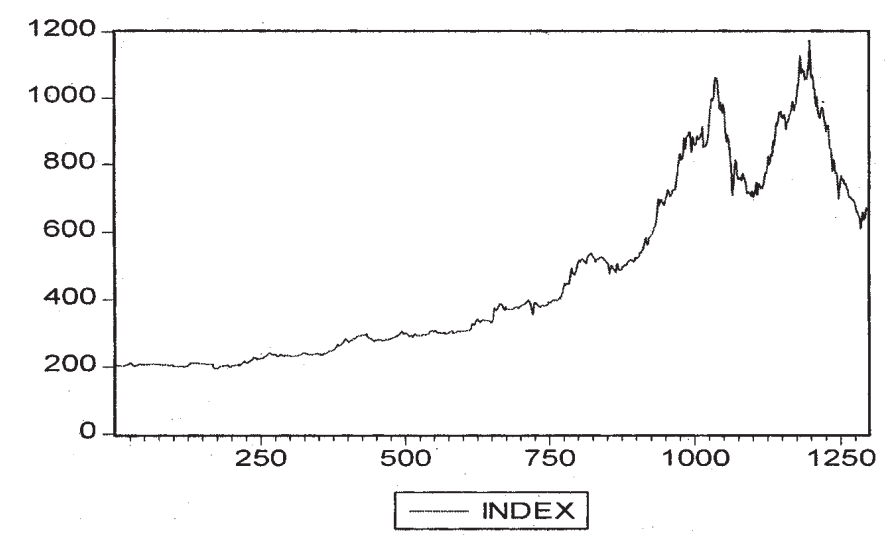

Appendix II: Autocorrelation of Daily NEPSE Return

\begin{tabular}{ccccc}
\hline Lag & AC & PAC & Q-Stat & Prob \\
\hline 1 & 0.377 & 0.377 & 184.15 & 0.000 \\
2 & 0.031 & -0.129 & 185.41 & 0.000 \\
3 & -0.051 & -0.019 & 188.80 & 0.000 \\
4 & 0.007 & 0.046 & 188.86 & 0.000 \\
5 & 0.037 & 0.015 & 190.68 & 0.000 \\
10 & 0.098 & 0.055 & 211.80 & 0.000 \\
15 & 0.016 & -0.014 & 238.78 & 0.000 \\
20 & 0.049 & 0.040 & 245.28 & 0.000 \\
25 & 0.009 & -0.018 & 268.48 & 0.000 \\
28 & -0.001 & 0.015 & 270.33 & 0.000 \\
29 & 0.000 & -0.019 & 270.33 & 0.000 \\
\hline
\end{tabular}

